Onkologe 2020 $\cdot 26: 311-316$

https://doi.org/10.1007/s00761-020-00735-4

Online publiziert: 24. Februar 2020

(c) Der/die Autor(en) 2020

Klaus Kraywinkel · Nina Buttmann-Schweiger

Zentrum für Krebsregisterdaten, Robert Koch-Institut, Berlin, Deutschland

\title{
Epidemiologie bösartiger Tumoren der Speiseröhre in Deutschland unter Berücksichtigung der histologischen Typen
}

\section{Einführung}

Bei den bösartigen Tumoren der Speiseröhre lassen sich als Hauptformen das Plattenepithel- (SCC) und das Adenokarzinom (Adeno-Ca.) identifizieren, die sich neben der Histologie auch ätiologisch und bezüglich ihrer Lokalisation unterscheiden: Während Plattenepithelkarzinome überwiegend im oberen und mittleren Drittel zu finden sind und mit Tabak und Alkoholkonsum ähnliche Risikofaktoren wie die Tumoren des hinteren Rachens und des Kehlkopfs aufweisen, sind die Adenokarzinome ganz überwiegend im unteren Drittel der Speiseröhre lokalisiert und werden mit den Karzinomen des Mageneingangs zu den bösartigen Tumoren des ösophagogastralen Übergangs (AEG I-III) zusammengefasst. Ursächlich werden hier vor allem Schleimhautveränderungen (Metaplasien) auf dem Boden einer Refluxerkrankung angenommen. Für Letztere hat vor allem Übergewicht eine prädisponierende Rolle, außerdem scheint eine Besiedlung mit Helicobacter pylori hier (im Gegensatz zum Magenkarzinom) protektiv $\mathrm{zu}$ wirken [1-3].

In Deutschland sind die altersstandardisierten Neuerkrankungsraten des Speiseröhrenkarzinoms seit der Jahrtausendwende für beide Geschlechter geringfügig angestiegen, während die Mortalitätsraten nahezu unverändert geblieben sind [1]. Bereits für die letzten 2 Jahrzehnte des 20. Jahrhunderts wurde aller- dings für einzelne europäische Länder vor allem bei den Männern ein deutlicher Anstieg der Adenokarzinome der Speiseröhre beschrieben, während die Inzidenz des SCC sich kaum verändert hat [4]. Mit Daten aus den bevölkerungsbezogenen Krebsregistern aus Hamburg, dem Saarland und dem Regierungsbezirk Münster wurde für die Jahre 1991-2012 bereits ein ähnlicher Trend in Deutschland gezeigt [5]. Im folgenden Beitrag wird erstmals eine Schätzung der bundesweiten Inzidenz der beiden Formen des Speiseröhrenkrebses vorgenommen, daneben werden die Verteilung der Tumorstadien und Überlebensraten für beide Formen verglichen.

\section{Datenquellen und Methoden}

Das Zentrum für Krebsregisterdaten führt seit 2009 jährlich die Krebsregisterdaten aller Bundesländer zusammen und schätzt deren Vollzähligkeit. Die Angaben zur bundesweiten Inzidenz der bösartigen Tumoren der Speiseröhre (ICD-10: C15) beruhen auf einer Schätzung der Neuerkrankungen, die für die noch bestehende Untererfassung in einzelnen Bundesländern korrigiert und Trendaussagen über einen längeren Zeitraum (ab 1999) ermöglicht [1]. Die Inzidenz der histologischen Formen wurde anhand des Anteils der jeweiligen Gruppen an allen erfassten Fällen (nach Alter, Geschlecht und Kalenderjahr) bestimmt. Die Gruppierung erfolgte anhand der Codierung entsprechend der 


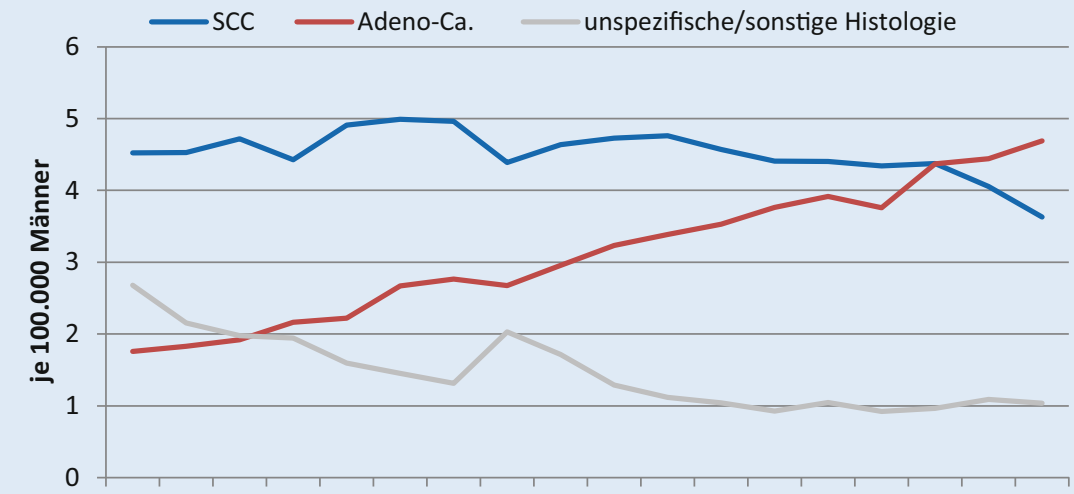

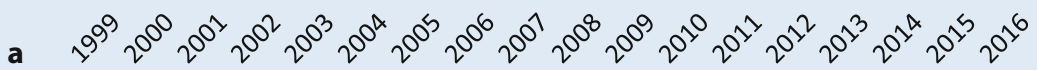

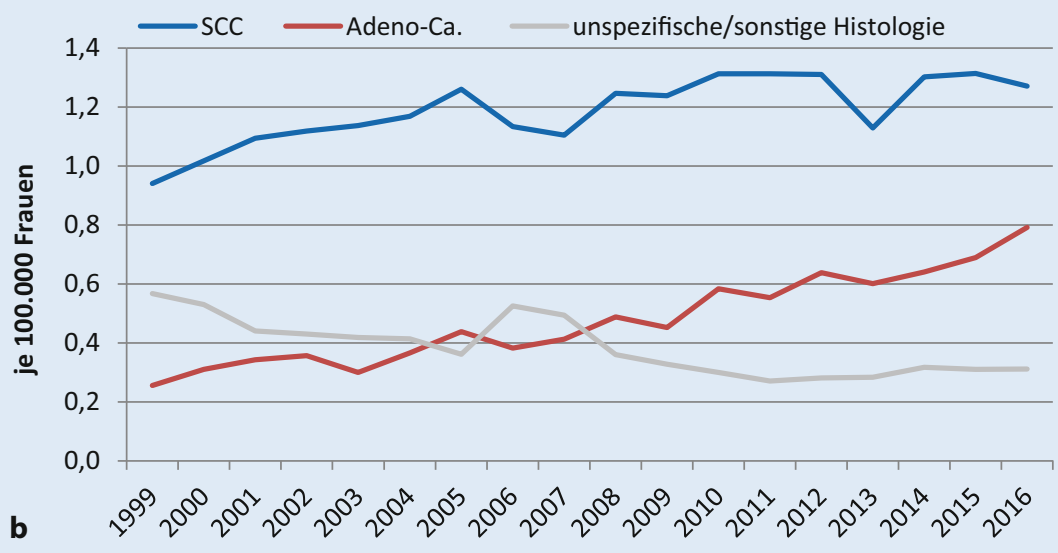

Abb. 1 ॥ Geschätzte altersstandardisierte Inzidenzrate des Ösophaguskarzinoms nach Histologie und Geschlecht (a Männer, b Frauen), Deutschland 1999-2016

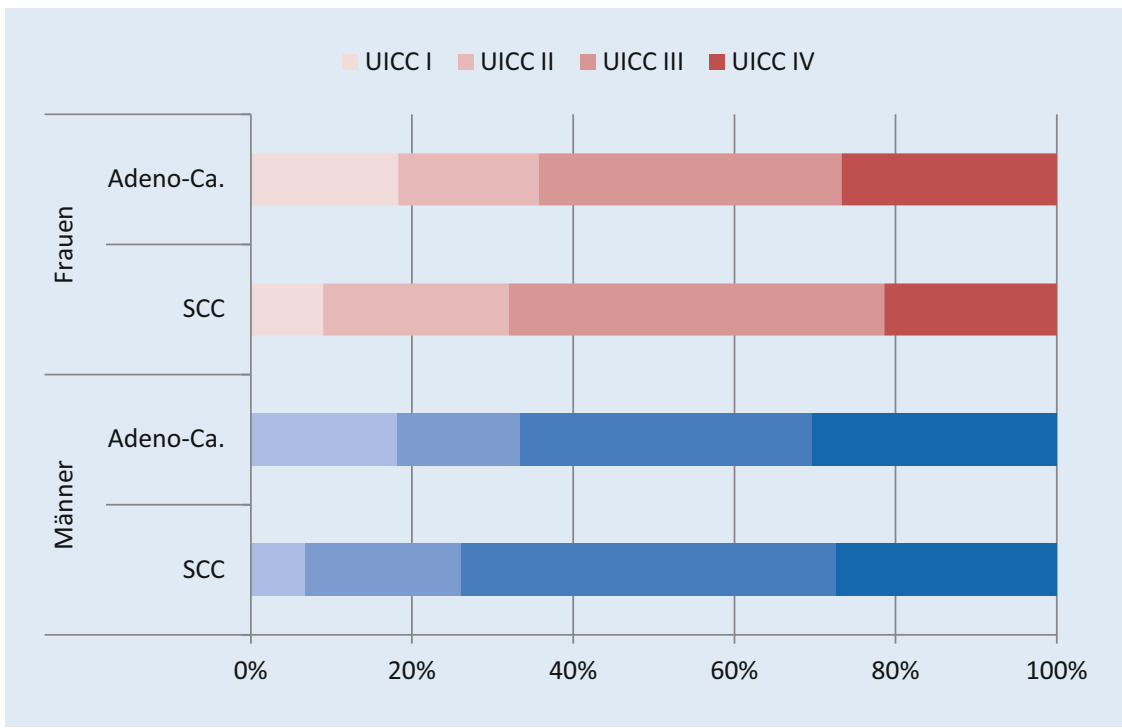

Abb. 2 ム Verteilung der Tumorstadien für das Ösophaguskarzinom nach Histologie und Geschlecht, Deutschland 2012-2016 dritten Fassung der Internationalen Klassifikation der Krankheiten für die Onkologie (ICD-O-3; SCC: 8050-8084, Adeno-Ca.: 8140-8384, 8401, 8480-8490, 8550-8552, 8570-8574, 8576). Unspezifische Histologien (8000-8035) und sonstige Formen (ca. $2 \%$ der Fälle) wurden in der Darstellung der Ergebnisse zusammengefasst. Zur Beurteilung der um demografische Veränderungen bereinigten Trends wurden altersstandardisierte Inzidenzraten nach altem Europastandard berechnet. Die Darstellung der Verteilung der Tumorstadien bei Erstdiagnose für die Jahre 2012-2016 erfolgte nach der 7. Auflage der Klassifikation maligner Tumoren der International Union Against Cancer (UICC).

Relative Überlebenswahrscheinlichkeiten wurden für den Zeitraum 20112015 nach der Periodenmethode berechnet [6]. Diese bilden die krebsbedingte Sterblichkeit ab, indem der Quotient aus dem beobachteten Überleben der Betroffenen und dem erwarteten Überleben in der allgemeinen Bevölkerung gleichen Alters und gleichen Geschlechts gebildet wird. Als Berechnungsgrundlage der Erwartungswerte für das Überleben der allgemeinen Bevölkerung werden die Periodensterbetafeln des Statistischen Bundesamts genutzt [7]. Für die Berechnung wurden Daten aus acht Krebsregistern herangezogen, welche die in „Krebs in Deutschland“ beschriebenen Qualitätskriterien erfüllen [1]. Nur über Todesbescheinigung identifizierte Fälle (DCO, zuletzt ca. 9\%) wurden bei den Auswertungen zum „survival“ wegen fehlenden Diagnosedatums ausgeschlossen, bei allen anderen Analysen jedoch berücksichtigt. Sie bildeten die Mehrzahl der Fälle mit fehlender Histologie.

\section{Ergebnisse}

Die geschätzte Zahl bösartiger Speiseröhrentumoren in Deutschland stieg von 1999 bis 2016 bei Frauen von 1120 auf 1740 und bei Männern von 4050 auf 5540 an. Die altersstandardisierten Inzidenzraten für das Adeno-Ca. lagen zuletzt bei beiden Geschlechtern mehr als doppelt so hoch wie um die Jahrtausendwende, für die SCC waren dagegen nur geringfügige Veränderungen $\mathrm{zu}$ verzeichnen. 
Hier steht eine Anzeige.

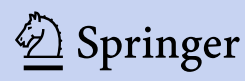


Rückläufig war für beide Geschlechter die Rate der unspezifischen bzw. sonstigen Histologien (• Abb. 1). Für das Jahr 2016 errechneten sich daraus insgesamt rund 3340 neu diagnostizierte Adenokarzinome der Speiseröhre (davon 600 bei Frauen) und 3030 Plattenepithelkarzinome ( 870 bei Frauen).

Das mediane Erkrankungsalter betrug bei Männern für beide Formen des Ösophaguskarzinoms zuletzt 67 Jahre, Frauen erkrankten mit 70 (SCC) bzw. 73 Jahren (Adeno-Ca.) im Mittel einige Jahre später.

In $56 \%$ aller erfassten Fälle waren die Angaben zum Tumorstadium ausreichend für eine Zuordnung der Stadien I-IV. Der Anteil sowohl früher (UICC I) als auch später Stadien (UICC IV) lag bei den Adeno-Ca. höher als bei den SCC, für beide Formen war die Stadienverteilung für Frauen etwas günstiger als für Männer (• Abb. 2).

Die relativen 5-Jahres-Überlebensraten lagen für das Adeno-Ca. bei 29\% und für das SCC bei $17 \%$, für Letzteres war die Prognose bei Frauen etwas besser als bei Männern (• Abb. 3). Abb. 4 zeigt die höheren Überlebensaussichten beim Adeno-Ca. gegenüber dem SCC vor allem im Stadium I. Im Vergleich zur Periode 2001-2005 hat sich das relative 5-Jahres-Überleben bei den AdenoCa. um 4,1 und bei den SCC um 2,0\% verbessert (ohne Abbildung).

\section{Diskussion}

Die vorliegenden Ergebnisse bestätigen auch für Deutschland den für andere europäische Länder und einige deutsche Regionen bereits für die 1980er- und 1990er-Jahre gezeigten Anstieg der Inzidenz von Adenokarzinomen der Speiseröhre $[4,5]$. Dieser Anstieg scheint sich weiter fortzusetzen, inzwischen bilden die Adenokarzinome bei den Männern, aber auch insgesamt die Mehrzahl der bösartigen Speiseröhrentumoren. Berücksichtigt man den rückläufigen Anteil der Fälle mit unspezifischen Angaben zur Histologie, ist für das Plattenepithelkarzinom der Speiseröhre zumindest bei den Männern zuletzt von einem Rückgang der Inzidenz auszugehen. Dies entspricht der Entwicklung bei

Onkologe 2020 $26: 311-316$ https://doi.org/10.1007/s00761-020-00735-4

(c) Der/die Autor(en) 2020

\section{K. Kraywinkel · N. Buttmann-Schweiger}

\section{Epidemiologie bösartiger Tumoren der Speiseröhre in Deutschland unter Berücksichtigung der histologischen Typen}

\section{Zusammenfassung}

Im vorliegenden Beitrag werden erstmals für Deutschland Inzidenz- und Überlebensraten für die beiden wesentlichen Formen des Speiseröhrenkarzinoms präsentiert, basierend auf den Daten der bevölkerungsbezogenen Krebsregister. Für beide Geschlechter ist seit 1999 ein Anstieg der altersstandardisierten Inzidenzraten der Adenokarzinome zu erkennen, während die Raten für Plattenepithelkarzinome (SCC) bei Frauen stagnieren und bei Männern zuletzt rückläufig sind. Zuletzt (2016) überwogen in Deutschland die Adenokarzinome mit 3340 Fällen gegenüber 3030 Plattenepithelkarzinomen. Knapp jede fünfte (Adeno-Ca.) bzw. jede dritte Neuerkrankung (SCC) trat bei Frauen auf. Die
Verteilung der Tumorstadien war für beide Formen bei Frauen etwas günstiger als bei Männern, für das Plattenepithelkarzinom lagen auch die relativen Überlebensraten für Frauen höher. Auch unabhängig vom Tumorstadium wiesen Adenokarzinome eine etwas günstigere Prognose auf, dies galt vor allem im frühen Stadium (UICC I). Über 10 Jahre haben sich die relativen 5-JahresÜberlebensraten um 4,1 (Adeno-Ca.) bzw. 2,0 Prozentpunkte (SCC) verbessert.

\section{Schlüsselwörter}

Plattenepithelkarzinom · Adenokarzinom . Inzidenz · Überleben · Krebsregister · Deutschland

\section{Epidemiology of esophageal malignancies in Germany with regard to histological subtypes}

\section{Abstract}

The following contribution is the first to present incidence and survival rates for the two major types of esophageal malignancies, based on population-based cancer registry data. For both gender, age-standardized incidence rates for adenocarcinoma have increased since 1999, while those for squamous cell carcinoma (SCC) of the esophagus were stable for women and recently declining for men. In 2016, 3340 people in Germany were diagnosed with adenocarcinoma and 3030 with squamous cell carcinoma. Approximately one in five adenocarcinoma and every third SCC were diagnosed in women. The distribution of tumor stages was slightly more favorable for both subtypes for women, and the relative survival rates for SCC were also higher for women. Adenocarcinoma had a more favorable prognosis compared to SCC, especially in stage I tumors. Over the last decade, the relative 5 -year survival improved by $4.1 \%$ for adenocarcinomas and $2.0 \%$ for squamous cell carcinomas.

\section{Keywords}

Squamous cell carcinoma - Adenocarcinoma . Incidence · Survival · Cancer registries . Germany anderen tabakassoziierten Tumoren, insbesondere dem Bronchialkarzinom [1]. Die Ergebnisse deuten auf eine zunehmende Bedeutung von Übergewicht und Reflux in der Ätiologie dieser Erkrankung hin, fast die Hälfte der Adenokarzinome der Speiseröhre in Deutschland kann auf Übergewicht zurückgeführt werden [8]. Daneben kann der Rückgang der Besiedlung von Helicobacter pylori in der Magenschleimhaut eine Rolle spielen, allerdings dürfte der risikoerhöhende Einfluss dieses Bakteriums auf die Inzidenz des Magenkarzinoms den wahrscheinli- chen protektiven Effekt in der Speiseröhre deutlich überwiegen [2, 3].

Die Daten aus den deutschen Krebsregistern bestätigen ferner eine leichte Verbesserung der Überlebensraten, die bereits mit früheren Daten aus Deutschland bzw. Europa gezeigt wurde [9-11]. Die nach Histologie stratifizierten Ergebnisse zeigen, dass diese nur zum Teil durch eine Verschiebung der Inzidenz in Richtung der mit einer etwas besseren Prognose verbundenen Adenokarzinome erklärt werden kann. 


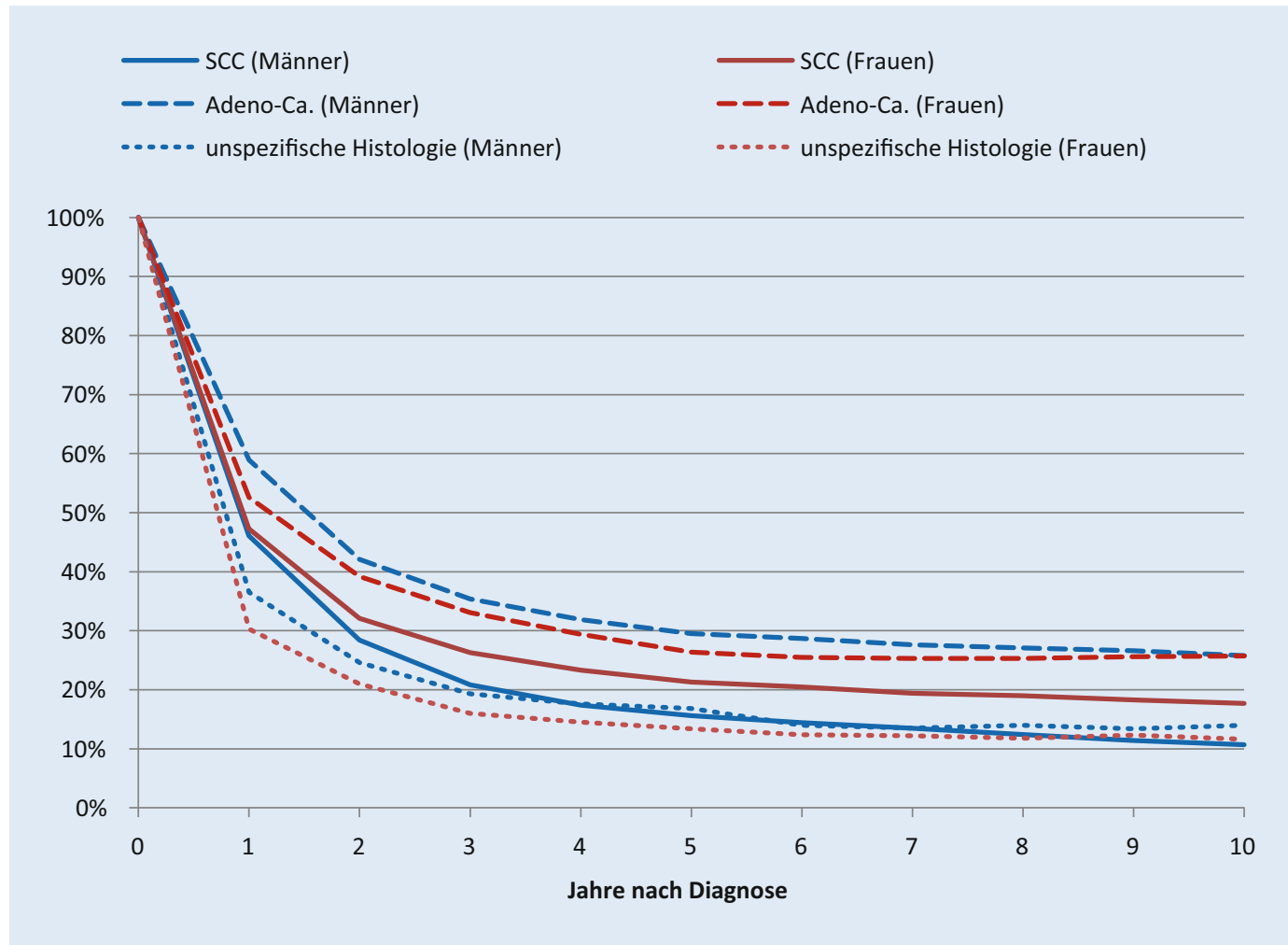

Abb. $3<$ Relatives Überleben bis 10 Jahre nach Diagnose von Speiseröhrenkarzinomen nach Histologie und Geschlecht (Deutschland, Periode 2011-2015)

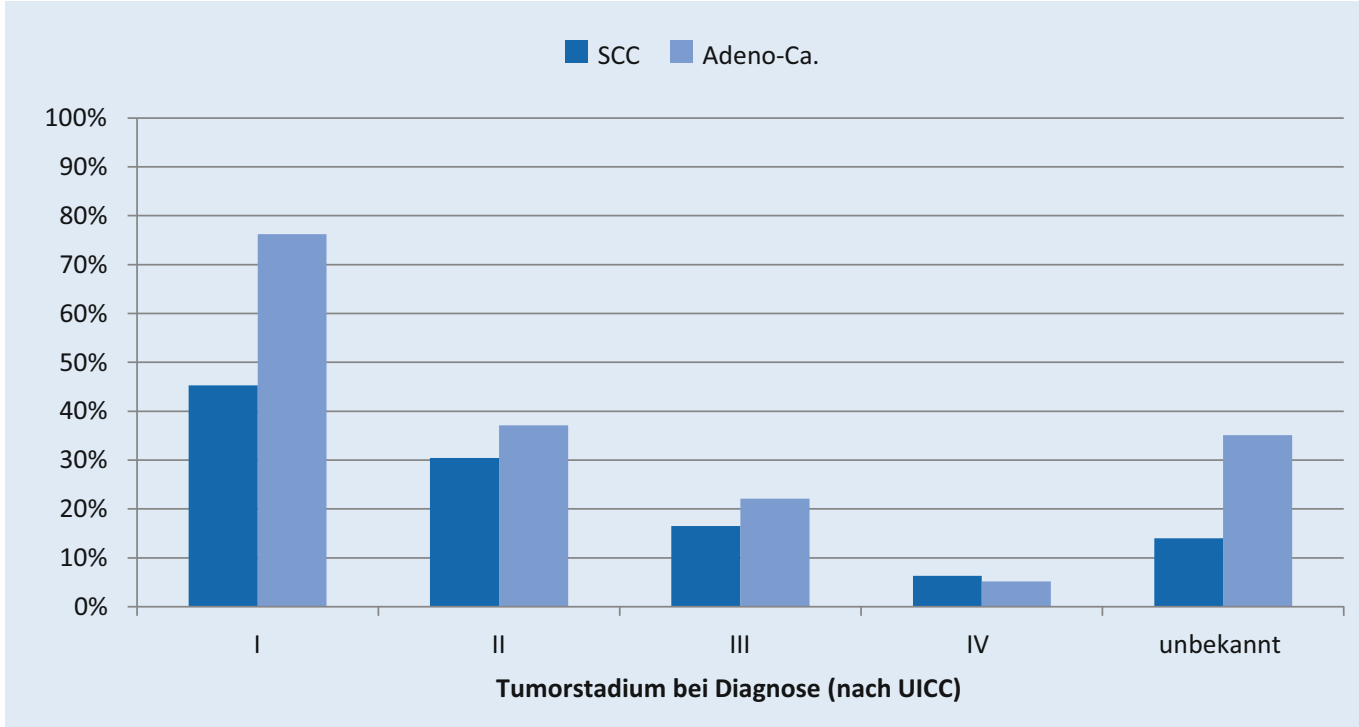

Abb. $4<$ Relatives 5-Jahres-Überleben bei Speiseröhrenkarzinomen nach Histologie und Tumorstadium bei Diagnose (Deutschland, Periode 2011-2015)

\section{Fazit}

Eine Differenzierung der Auswertungen bevölkerungsbezogener Krebsregister für die beiden wesentlichen Formen des Speiseröhrenkarzinoms trägt zum Verständnis der Epidemiologie dieser Erkrankung bei.

\section{Korrespondenzadresse}

\section{Dr. Klaus Kraywinkel}

Zentrum für Krebsregisterdaten, Robert KochInstitut

Postfach 650261, 13302 Berlin, Deutschland

KraywinkelK@rki.de

Funding. Open Access funding provided by Projekt DEAL.

\section{Einhaltung ethischer Richtlinien}

Interessenkonflikt. K. Kraywinkel und N. ButtmannSchweiger geben an, dass kein Interessenkonflikt besteht.

Für diesen Beitrag wurden von den Autoren keine Studien an Menschen oder Tieren durchgeführt. Für die aufgeführten Studien gelten die jeweils dort angegebenen ethischen Richtlinien. 
Open Access. Dieser Artikel wird unter der Creative Commons Namensnennung 4.0 International Lizenz veröffentlicht, welche die Nutzung, Vervielfältigung Bearbeitung, Verbreitung und Wiedergabe in jeglichem Medium und Format erlaubt, sofern Sie den/die ursprünglichen Autor(en) und die Quelle ordnungsgemäß nennen, einen Link zur Creative Commons Lizenz beifügen und angeben, ob Änderungen vorgenommen wurden.

Die in diesem Artikel enthaltenen Bilder und sonstiges Drittmaterial unterliegen ebenfalls der genannten Creative Commons Lizenz, sofern sich aus der Abbildungslegende nichts anderes ergibt. Sofern das betreffende Material nicht unter der genannten Creative Commons Lizenz steht und die betreffende Handlung nicht nach gesetzlichen Vorschriften erlaubt ist, ist für die oben aufgeführten Weiterverwendungen des Materials die Einwilligung des jeweiligen Rechteinhabers einzuholen.

Weitere Details zur Lizenz entnehmen Sie bitte der Lizenzinformation auf http://creativecommons.org/ licenses/by/4.0/deed.de.

\section{Literatur}

1. Robert Koch-Institut, Gesellschaft der epidemiologischen Krebsregister in Deutschland e. V. (2019) Krebs in Deutschland für 2015/2016. 12. Ausgabe. Robert Koch-Institut, Gesellschaft der epidemiologischen Krebsregister in Deutschland e. V., Berlin

2. Fischbach LA, Nordenstedt $H$, Kramer JR et al. (2012) The association between Barrett 's esophagus and Helicobacter pylori infection: a meta-analysis. Helicobacter 17(3):163-175

3. International Agency for Research on Cancer (Hrsg) (2009) IARC Monographs on the Evaluation of Carcinogenic Risks to Humans. A Review of Human Carcinogens. Part B: Biological Agents. Volume 100 B. IARC, Lyon

4. Castro C, Bosetti C, Malvezzi M, Bertuccio P, Levi F, Negri E, La Vecchia C, Lunet N (2014) Patterns and trends in esophageal cancer mortality and incidence in Europe (1980-2011) and predictions to 2015. Ann Oncol25(1):283-290.https://doi.org/ 10.1093/annonc/mdt486

5. Hoeppner J, Glatz T, Claus R, Fischer A, Kraywinkel K, Brunner T (2017) Das Adenokarzinom des Ösophagus - Bestandsaufnahme einer drastisch zunehmenden Erkrankung. Dtsch Med Wochenschr 142(19):1453-1460. https://doi.org/10.1055/ s-0043-115777

6. Brenner $\mathrm{H}$ et al (2004) Period analysis for 'up-todate' cancer survival data: theory, empirical evaluation, computational realisation and applications. EurJ Cancer 40(3):326-335

7. Destatis Amtliche Todesursachenstatistik des Statistischen Bundesamtes. www.gbe-bund.de. Zugegriffen: 16.02.2020

8. Wienecke A, Neuhauser H, Kraywinkel K, Barnes B (2010) (2018) Cancers Potentially Preventable through Excess Weight Reduction in Germany in. Obes Facts 11(5):400-412. https://doi.org/10. 1159/000490150 (Epub 2018 Oct 17)

9. Hiripi E, Jansen L, Gondos A, Emrich K, Holleczek B Katalinic A, Luttmann S, Nennecke A, Brenner H, GEKID Cancer Survival Working Group (2012) Survival of stomach and esophagus cancer patients in Germany in the early 21 st century. Acta Oncol 51(7):906-914
10. Anderson LA, Tavilla A, Brenner H, Luttmann S, Navarro C, Gavin AT, Holleczek B, Johnston BT, Cook MB, Bannon F, Sant M, the EUROCARE-5 Working Group (2015) Survival for oesophageal, stomach and small intestine cancers in Europe 1999-2007: Results from EUROCARE-5. Eur J Cancer 51:2144-2157

11. Launoy G, Bossard N, Castro C, Manfredi S, the GRELL EUROCARE-5 Working Group (2017) Trends in net survival from esophageal cancer in six European Latin countries: results from the SUDCAN population-based study. Eur J Cancer Prev 26:24-31

\section{ONKO-Internetportal}

Rückblick Deutscher Krebskongress

Der Deutsche Krebskongress (DKK) in Berlin stand in diesem Jahr unter dem Motto „informativ. innovativ. integrativ. Optimale Versorgung für alle". Rund 10.000 Teilnehmende aus Wissenschaft, Ärzteschaft, Gesundheitswesen, Politik und Verwaltung tauschten sich vor Ort über die jüngsten Fortschritte in der Krebsmedizin aus.

Journalistisch begleitet wurde der DKK vom Redaktionsteam des ONKO-Internetportals in Kooperation mit der Deutschen Krebsgesellschaft. Auf dem Onlineportal informieren Experten in Video-Interviews und Expertenrunden über die auf dem Kongress präsentierten Erkenntnisse und damit verbundenen Herausforderungen in den unterschiedlichen Fachgebieten. Im Expertengespräch zum fortgeschrittenen hepatozellulären Karzinom" (HCC) werden u. a. die Kriterien für Therapiewahl und Sequenz im Kontext zielgerichteter Erst- und Zweitlinientherapien diskutiert. Bei den Experteninterviews liegt der Fokus auf Innovationen im Bereich der gastrointestinalen Tumoren, beim metastasierten Kolorektalkarzinom und beim nicht-kleinzelligen Lungenkarzinom. Zur Sprache kommen außerdem Langzeitbeobachtung und Remissionsqualität beim Nierenzellkarzinom sowie die palliativmedizinische Versorgung bei Krebserkrankungen und die Versorgung junger Menschen mit Krebs. Interessierte Ärzte finden eine kompakte Zusammenfassung der wichtigsten Kongressereignisse unter www.krebsgesellschaft.de/dkk2020.

Quelle: ONKO-Internetportal in

Kooperation mit der Deutschen Krebsgesellschaft e.V. (DKG), www.krebsgesellschaft.de 